\title{
A STUDY ON STATISTICAL RECURRENCE OF SOME DISTINGUISHING FEATURES OF HANDWRITINGS AND SIGNATURES
}

\author{
M. Pradhan, B. P. Bhusal, P. Bhattarai, P. M. Shakya, Bashudev K.C., M. Bajracharya \\ National Forensic Science Laboratory, Lalitpur, Nepal
}

\begin{abstract}
A hundred of different cases with questioned handwritings and signatures of a hundred different individuals written on the time interval from one year to sixty-one years were studied and the twenty five distinguishing characteristics were found to recur in different extents. A total of 764 points were noted with average of 7 to 8 points per case and statistics of recurrence of the same characteristics was built up so as to explain the most recurring and least recurring characteristics of the writings and signatures that resembled to assert the common origin even after the long interval of time. Amidst the variables, the 'design of letters and specific patterns' was found to be the most recurring whereas 'tie formation ' and 'position, shape and size of dot (.)'were found to be the least.
\end{abstract}

Keywords: Handwriting; Signature; Rhythm; Stroke; Recurrence; Synchronization.

\section{INTRODUCTION}

Forensic document examination involves the examination and comparison of handwriting and/or signatures. In questioned document cases there are many instances when it is not possible for the expert to take specimen handwriting and/or signature. There may also be a significant time gap between the admitted handwriting and/or signature and the questioned handwriting and/or signature. Handwriting is a complex perceptual motor task which is an acquired skill. Handwriting identification depends upon the various extrinsic and intrinsic factors. Extrinsic factors include environmental conditions, position and style of holding pen and paper, etc. Intrinsic factors include health, mental stability, etc. ${ }^{1}$ Handwriting identification also is a discriminatory process that is derived from the comparison of writing habits, and an evaluation of the significance of their similarities or differences. $^{2}$

Writing is the process of assigning symbols to abstract ideas as well as concrete things that carry some meaning which is well accepted within the community the writing claims to belong to. It is the conscious plus subconscious attempt to produce letters or symbols to represent ideas, feelings, facts or, say, the things. In the process of hand printing, the muscular and nervous systems are naturally well synchronized such that unique and uniform letters and symbols are produced within the range of natural variation. Such uniqueness becomes the identity of the writer knowingly or unknowingly.

Human brain and muscular mechanics have some sorts of flexibility that can't produce exactly identical letters. There is some variation in writing due to some external environmental factors, health, mental conditions etc. However, such variations become easily distinguishable within some narrow range and can be averaged by increasing volume of samples.

Time is one of the major factors for all writers of whatever skill that intrinsically modifies the writing pattern and the designing of symbols. It is the most conspicuous and predictable factor that changes the various aspects of the writings. Most of the writings written in a long interval of time are arduous to be distinguished as well. However, there are several inherent characteristics in writings that are unique, distinctive, habitual and individualized that redeem questions with the successful resolution and rational solutions.

Writing skill of a person is a habitual phenomenon that persists even for ages. Though some visible differences may appear in some writings by same writer over ages misleading the examiners to assert their common origin, these can be authenticated to be of same origin by taking consideration over their unique writing habit which reappears even after some decades. Such individualization becomes so natural that it can be supported by several intrinsic writing behaviors that have been considered here as the parameters of writing comparison.

Free, natural writing is the almost unconscious visible expression of firmly established muscular habits based on fixed mental impressions of certain forms or outlines. These muscular habits, as well as the mental patterns, differ in a marked manner in different individuals and this variation radically affects the visible result. The forger usually acts on the false assumption that all writings are produced in the same manner and differ only in design of letter. ${ }^{3}$ It is very challenging yet unavailing job to imitate the job of writing of one person by the other. It is because of some uniqueness in writing just like letter designing and designing of pattern, slope, spacing, pen throw etc. that define the highly individualized features of writings and even if one attempts to copy the style of writing considering all the features inherent to those writings, the failure of synchronization between the nervous system and muscular system of hands while adoption of novel styles tends to return to and repeat the original patterns that makes all the endeavors go in vain. In other words, nobody can imitate the writings of others identically. 


\section{METHODOLOGY}

In this study, meticulous comparative analysis of a hundred real cases resolved by National Forensic Science Laboratory of Nepal concerning questioned handwritings and signatures at different dates, with one to sixty one years' gap between questioned writings and sample writings, was carried out and the spectrum of twenty five different basic characteristics focused for the possible forgery was built up. All the related technical reports were processed to build up statistics on the basis of recurrence of the several characteristics in the individual reports. Then comparative statistical analysis was carried out to reach the conclusion.

\section{DATA, INTERPRETATION AND RESULT}

Comparative study of questioned writings with the sample ones was carried out and counted the recurrence of twenty five distinct characteristics while reporting the cases. It birthed to the following statistics:

\begin{tabular}{|c|c|c|c|}
\hline S.N. & Distinguishing characters & No. of recurrence & \% Occupancy \\
\hline 1 & Study of tie formation & 06 & 0.78 \\
\hline 2 & Shape and size of sign & 07 & 0.92 \\
\hline 3 & Tremors and hesitation & 08 & 1.05 \\
\hline 4 & Loop structure & 08 & 1.05 \\
\hline 5 & Speed & 10 & 1.31 \\
\hline 6 & Rhythm & 15 & 1.96 \\
\hline 7 & Pressure & 25 & 3.27 \\
\hline 8 & Design of $f, \mathrm{~T}_{\text {, }}$, etc. & 26 & 3.40 \\
\hline 9 & Pen throw condition & 27 & 3.53 \\
\hline 10 & Initial and following letters & 48 & 6.28 \\
\hline 11 & Comparision of initial, middle and last stroke variation & 54 & 7.33 \\
\hline 12 & Slope & 67 & 8.77 \\
\hline 13 & Designing of letters and specific patterns & 139 & 18.19 \\
\hline 14 & Spacing & 66 & 8.63 \\
\hline 15 & Ending and ending points of strokes & 51 & 6.68 \\
\hline 16 & Position of $f, \mathrm{t}_{\text {, }}$, etc. & 46 & 6.02 \\
\hline 17 & Shape of letters & 27 & 3.53 \\
\hline 18 & Size & 26 & 3.40 \\
\hline 19 & Diga & 25 & 3.27 \\
\hline 20 & Pen pause and pen lift & 25 & 3.27 \\
\hline 21 & Base line study & 17 & 2.22 \\
\hline 22 & Alignment & 13 & 1.70 \\
\hline 23 & Size and shape of underline & 13 & 1.70 \\
\hline 24 & Ink deposition & 09 & 1.18 \\
\hline 25 & Position, shape and size of '.' & 06 & 0.78 \\
\hline
\end{tabular}

Figure: Data table 


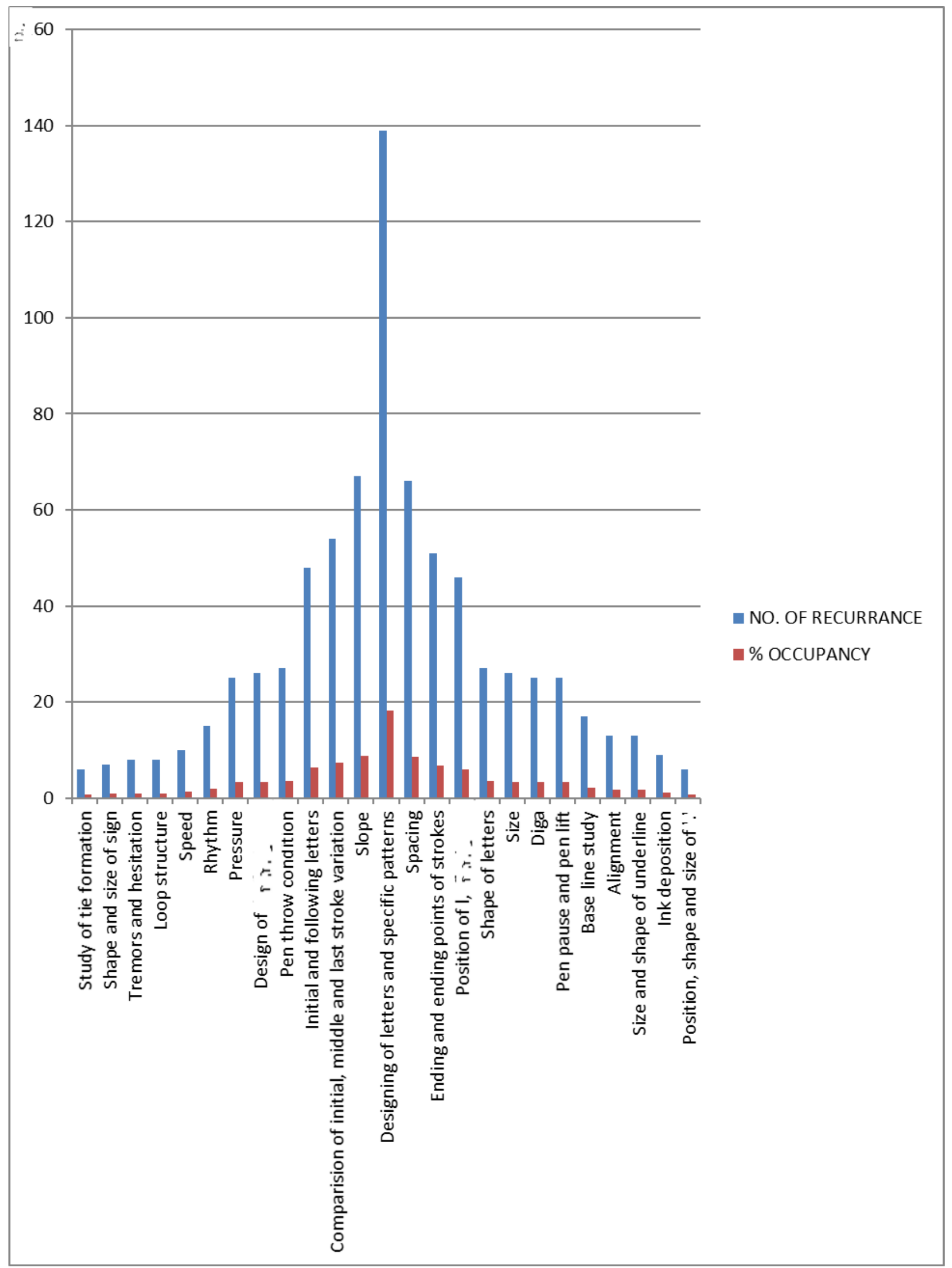

Figure : Bar graph representation of data table 


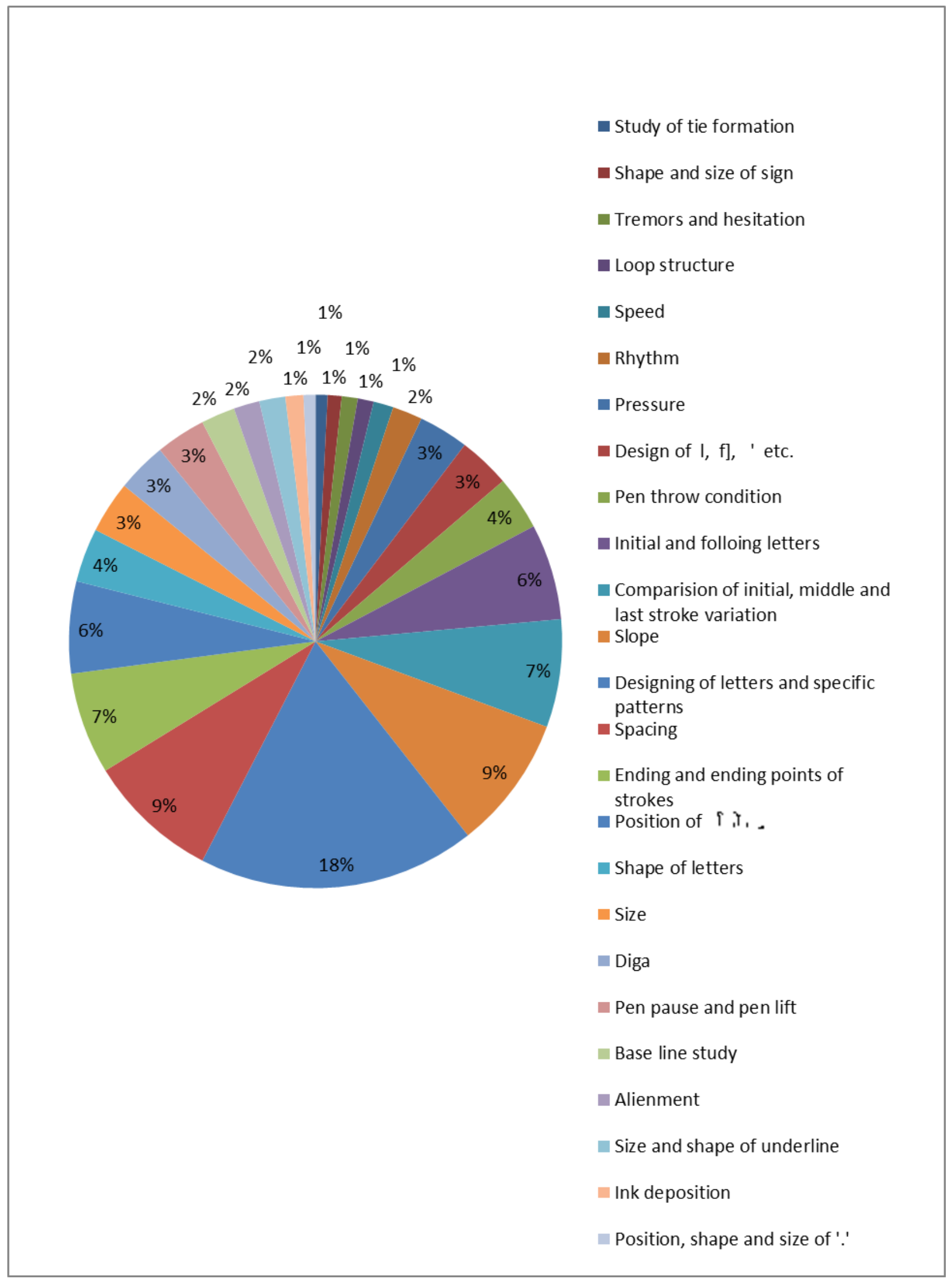

Figure : Pie chart representation of the data table 
Here, the maximum repeating characteristics focused to compare the questioned writings with the sample writings to prove common origin was found to be 'designing of letters and specific patterns' with frequency of 139 which was followed by 'slope' with frequency of 67 and 'spacing' with frequency of 66 , followed by many others shown in the table above. It means that out of 764 points noted in the 100 cases, 139 points (i.e. $\approx 18.19 \%$ ) were about the design of letters and patterns that focused on how some specific letters and patterns were formed by the people considered as their unique characteristics of writing that remained unchanged over a long period of time. In comparison to others, this trait was found to be most distinct point and was the major foundation for drawing the conclusion.

The next prominent characteristics was 'slope', like slope of patterns, rhythms etc. It occupied 67 points $(\$ 8.77 \%)$ of all the points focussed on while reporting those cases. Similarly, 'spacing' of letters and words occupied 66 points $(\approx 8.63 \%)$. Theses are the features most often emphasized to find the similarities and differences, if any, between the questioned and sample writings to verify the common source. Other $7.33 \%$ of the points were related to 'comparison of initial, middle and last stroke variation' and $6.68 \%$ of points were related to how ending and ending points of strokes were formulated in writing. Further, the mostly recurred characteristics were 'Design

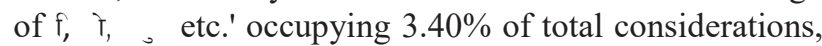
but this one feature is mostly language-specific which may not be any significant characteristics to English and many other languages. The same weight is carried by 'size' which signifies for the size of letters, symbols etc. 'Diga' is another language-specific, yet important distinguishing feature of handwritings and signatures. The other one of the most recurring feature is 'pen-throw ' $(\approx 3.93 \%)$ and 'pen pause and pen lift' also carries a significant weight while distinguishing writings. 'Shape of letters' $(\approx 3.53 \%)$ is based on whether the letters are circular or angular and is one of the very intrinsic form.

\section{DISCUSSION AND CONCLUSION}

The mean recurrence of the characteristics of the above mentioned data table is 30.56 but it's standard deviation (S.D.) is 28.79. It means the categorical data is more scattered from the mean value. Even if we took the range of $\bar{X}($ mean $) \pm \sigma\left(S . D_{x}\right)$ and $\bar{X}($ mean $) \pm 2 \sigma\left(S . D_{x}\right)$, it still excludes the most recurring characteristics 'the design of letters and specific patterns'. Moreover, the least recurring characteristics may be statistically less significant, but these become decisively significant in many cases and their contribution cannot be overlooked. Most importantly, all these characteristics are not found in the same writing and may not be encompassed by the writing skill of a single writer and so it becomes insubstantial to statistically generalize the writing characteristics of different persons in whatever time gap by the use of any standard statistical tool.

Under some time gap of some years to decades, the characteristic features of handwritings and signatures like designing of letters and specific patterns, slope, spacing, comparison of strokes and stroke variations, initial and following letters etc. remain unchanged and most of the questioned documents concerned to handwritings and signatures can be compared to the sample documents of the similar type to ascertain their common origin on the basis of the distinguishing characteristics mentioned in the table above and the priority of selection of such characters can most often be from most recurring to the least recurring one.

\section{ACKNOWLEDGEMENT}

Our best appreciation goes to Mr. Roshan Giri, Miss Manisha Rai and Mr. Bikash Gupta for their continuous dedication to ease our work and special thanks goes to Mr. Rameshwar Poudel for his computer assistance.

\section{REFERENCES}

1. Huber RA, Headrick AM. Handwriting Identification: Facts and Fundamentals. CRC Press LLC, 1999.

2. Aneja P., A Comparative Study of Peculiarities of Asynchronous Handwriting with Time Gap,International Journal of Multidisciplinary Research and Development,2016,Vol. 3, p.p.-277-279.

3. Osborn AS, Questioned Document, Albany NY. Boyd Print Co. $2^{\text {nd }}, 1929$. 